\title{
Prenatal diagnosis of 22q11.2 deletion when ultrasound examination reveals a heart defect
}

Shelina Manji, MD, Jacquelyn R. Roberson, MD, Anne Wiktor, BSMT(ASCP), Subodh Vats, MD, Peggy Rush, MS, Suzanne Diment, RN, BA, and Daniel L. Van Dyke, PhD

Purpose: The incidence of 22q11.2 deletion syndrome is approximately 1 in 5,000 births, and accounts for $5-30 \%$
of all heart defects, making it one of the more common genetic conditions in the population. Methods: We
employed fluorescence in situ hybridization (FISH) to study the incidence of $22 q 11.2$ deletions in fetuses with
cardiac anomalies detected on ultrasound examination. Results: Of 64 cases, 18 had visible chromosome
anomalies. FISH testing for $22 q 11.2$ deletion was performed on the remaining 46 cases, and five exhibited a
22q11.2 deletion. Three of the five had de novo deletions, one was maternally inherited, and one family declined
testing. Conclusion: FISH analysis for 22q11.2 deletion should be performed on all fetuses with cardiac defects
(excluding hypoplastic left heart and echogenic focus) and a normal G-banded karyotype. Genetics in Medicine, 2001:3(1):65-66.

Key Words: chromosome deletion, 22q11.2 deletion syndrome, prenatal diagnosis, cytogenetics, heart defect

Studies of liveborn infants suggest that the 22q11.2 deletion syndrome occurs in approximately 1 per 5,000 births..$^{1,2}$ Most cases are sporadic, though familial cases have been reported. Once classified as separate syndromes with overlapping features (DiGeorge, velocardiofacial, Shprintzen), a variety of conditions have been determined to result from the same chromosomal alteration. ${ }^{3}$ The clinical features associated with 22q11.2 deletion include conotruncal cardiac defects, thymic hypoplasia, cleft palate, hypocalcemia, learning difficulties, and abnormal faces characterized by retrognathia, a prominent nose with a squared nasal root, a deficient malar area, and a long face. More importantly, however, this syndrome accounts for $5-30 \%$ of all congenital heart defects. ${ }^{1,4}$ Because of the neonatal morbidity related to congenital heart defects, prenatal diagnosis of this syndrome has significant implications for the care of the infant and genetic counseling of the family.

Prenatal diagnosis is possible through the use of fluorescence in situ hybridization (FISH) studies. While there have been several reports in the literature of prenatal diagnosis of 22q11.2 deletion, there are little data addressing the incidence of the deletion among individuals with cardiac defects detected on prenatal ultrasound. One study identified a 22q11.2 deletion in 3 of $26(11.5 \%)$ pregnancies with a cardiac defect on fetal ultrasound and a negative family history. ${ }^{5}$ We report here the results of a study to examine the incidence of $22 \mathrm{q} 11.2 \mathrm{de}-$ letion in pregnancies in which congenital heart defects were identified by ultrasound examination.

\footnotetext{
From the Department of Medical Genetics, Henry Ford Health System, Detroit, Michigan. Daniel L. Van Dyke, PhD, Department of Medical Genetics, 2799 West Grand Boulevard, Detroit, MI 48202.

Received: October 11, 2000.

Accepted: October 27, 2000.
}

\section{PATIENTS AND METHODS}

The objective of this study was to investigate the incidence of $22 \mathrm{q} 11.2$ deletion syndrome in fetuses with cardiac anomalies noted on ultrasound, noncontributory family history, and normal karyotype. All cases referred to our laboratory from January of 1997 to May of 2000 were included.

If the request form for prenatal diagnosis noted a heart defect, the case was included in the study. The exceptions were hypoplastic left heart and echogenic focus (or foci) in the heart, since these anomalies are not known to be associated with 22q11.2 deletion. Cases referred for a positive family history of a heart defect were studied separately. If the request indicated ultrasound abnormalities, but not otherwise specified, we attempted to obtain additional information at the time of specimen submission.

If the routine karyotype analysis result was normal, FISH analysis was performed using the DGS/VCFS probe (Vysis, Inc.). This probe contains the regions TUPLE 1, D22S553, D22S609, and D22S942, which detect deletions of the band 22q11.2. If a chromosome 22 deletion was detected in the fetus, FISH analysis on the parents was requested to determine the inheritance pattern.

\section{RESULTS}

There were 723 women referred for prenatal cytogenetic diagnosis because congenital abnormalities were observed on ultrasound examination. The abnormalities included choroid plexus cysts, cystic hygroma, club feet, neural tube defect, omphalocele, cleft lip and palate, and cardiac defect.

Of the 723 cases, 64 had cardiac abnormalities (excluding hypoplastic left heart and echogenic focus). Of these, 18 had an 
Table 1

Chromosome 22q11.2 deletions identified prenatally

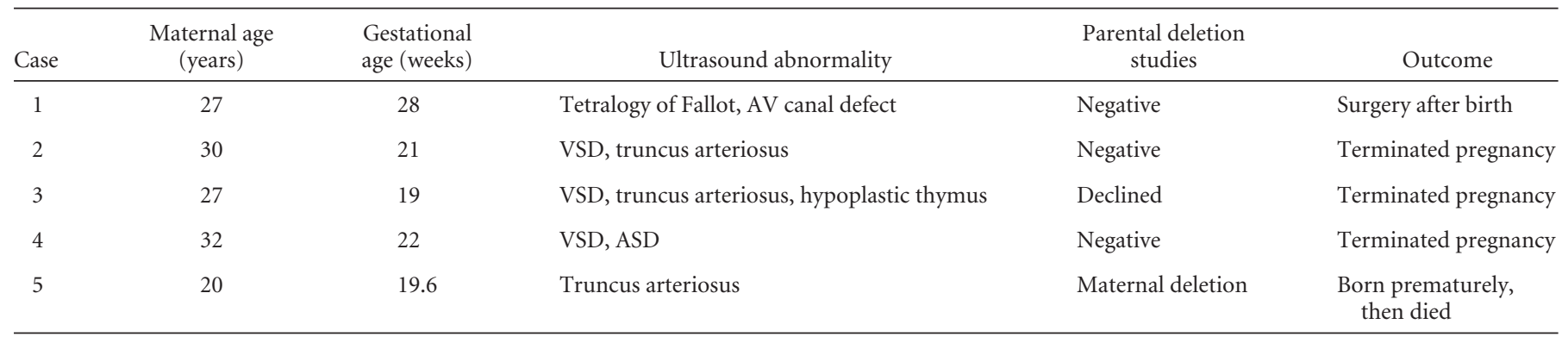

$\mathrm{AV}$, atriovenous; VSD, ventricular septal defect; ASD, atrial septal defect.

abnormal karyotype, including trisomy 13 (two cases), trisomy 18 (three cases), trisomy 21 (five cases), monosomy X (four cases), triploidy (two cases), and one case each of $46, \mathrm{XY}$, der (11)t(11;13) (q23;q14), and 46, XX, r(13) (p11q14.3). FISH analysis was not done on these 18 cases.

The remaining 46 cases had a normal karyotype by G-banding. FISH studies were completed on these 46 cases with normal karyotypes. Five of these (10.8\%) were found to be positive for the deletion (Table 1). Parental chromosome studies indicated that one case was maternally inherited, and three appeared to represent new mutations. In one case, the parents declined testing.

In all 10 cases referred for "family history of a heart defect," cytogenetics and FISH analysis were normal. These cases were not included in this study.

\section{DISCUSSION}

This study demonstrated a 22q11.2 deletion in about $11 \%$ of pregnancies that presented with conotruncal cardiac anomalies on fetal ultrasound, and that had a normal G-banded karyotype. The findings of our study are similar to those of Levy-Mozziconacci et al. ${ }^{5}$ Pooling the data from the two studies, 8 of $72(11 \%)$ pregnancies with cardiac anomalies detected on ultrasound were found to be positive for 22q11.2 deletion. Of the seven with family studies, five were found to be new mutations, and two were inherited.

Prenatal cytogenetic testing for heart defects is already indicated to identify aneuploidy and other visible chromosomal abnormalities. ${ }^{6}$ The present observations show that adjunctive testing for 22q11.2 deletion can provide many parents with additional information of value. Early diagnosis of 22q11.2 deletion offers the advantage of planning neonatal care, and decisions regarding cardiac repair can be made in a nonemergency context. Prenatal diagnosis also allows for genetic counseling regarding recurrence risk and identification of relatives at risk. In summary, we propose that FISH analysis for a $22 \mathrm{q} 11.2$ deletion is indicated when a heart defect is identified prenatally (excluding hypoplastic left heart or echogenic foci).

\section{Acknowledgments}

We greatly appreciate the support of the Cytogenetics Laboratory staff at Henry Ford Hospital in Detroit Michigan, and we thank Drs. Bsat, Goldfarb, Lazebnik, Powell, Quigg, Utter, Vettraino, and many other contributing physicians for their continued interest.

\section{References}

1. Wilson DI, Cross IE, Wren C, Scambler PJ, Burn J, Goodship J. Minimum prevalence of chromosome 22q11 deletions. Am J Hum Genet 1994;55:A169.

2. Wilson DI, Scrambler PJ. The chromosome 22q11 deletion syndromes. Appl Cytogent 1995;21:33-39.

3. Davidson A, Khandelwal M, Punnett H. Prenatal diagnosis of the 22q1 deletion syndrome. Prenat Diagn 1997;17:380-383.

4. Goldmuntz E, Driscoll D, Budarf M, Zackai E, McDonald-McGinn D, Biegel J, Emanuel B. Microdeletions of chromosome region $22 \mathrm{q} 11$ in patients with congenital conotruncal cardiac defects. J Med Genet 1993;30:807-812.

5. Levy-Mozziconacci A, Piquet C, Heurtevin PC, Philip N. Prenatal diagnosis of 22q11 microdeletion. Prenat Diagn 1997;17:1033-1037.

6. Ville YG, Nicolaides KH, Campbell S. Prenatal diagnosis of fetal malformations by ultrasound. In: Milunsky A, editor. Genetic disorders and the fetus, 4th ed. Baltimore: The Johns Hopkins University Press, 1998:750-811. 